\title{
DDoS Attacks Detection of Application Layer for Web Services using Information based Metrics
}

\author{
Nilesh A. Suryawanshi \\ Student \\ Department of Computer Science \\ JSPM'S ICOER, Pune, Maharashtra, India
}

\author{
S. R. Todmal \\ Faculty \\ Department of Computer Science \\ JSPM'S ICOER, Pune, Maharashtra, India
}

\begin{abstract}
Distributed Denial of Service attacks is major threats these days over internet applications and web services. These attacks moving forward towards application layer to acquire and waste maximum CPU cycles. By requesting resources from web services in huge amount using rapid fire of requests, attacker automated programs utilizes all the capability of processing of single server application or distributed environment application. The phases of the scheme implementation are user behavior monitoring and detection. In first phase by gathering the information of user behavior and calculating individual user's trust score will take place and Entropy of the same user will be calculated. Based on first phase, in detection phase, variation in entropy will be observed and malicious users will be detected. Rate limiter is also introduced to stop or downgrade serving the malicious users This paper presents the FAÇADE layer for detection and blocking the unauthorized user from attacking the system.
\end{abstract}

\section{General Terms}

Distributed Denial Services, Web Services, Trust Score

\section{Keywords}

Distributed Denial of Services, Entropy, Rate limiter, FAÇADE layer

\section{INTRODUCTION}

\subsection{Denial of Service Attacks}

A denial of service (DoS) attack is a malicious attempt to make a server or a network resource unavailable to users, usually by temporarily interrupting or suspending the services of a host connected to the Internet.

\subsection{Types of DoS Attacks}

The most common type of Denial of Service attack involves flooding the target resource with external communication requests. This overload prevents the resource from responding to legitimate traffic, or slows its response so significantly that it is rendered effectively unavailable.

Resources targeted in a DoS attack can be a specific computer, a port or service on the targeted system, an entire network, a component of a given network any system component. DoS attacks may also target human-system communications (e.g. disabling an alarm or printer), or human-response systems (e.g. disabling an important technician's phone or laptop).

DoS attacks can also target tangible system resources, such as computational resources (bandwidth, disk space, processor time); configuration information (routing information, etc.); state information (for example, unsolicited TCP session resetting). Moreover, a DoS attack can be designed to: execute malware that maxes out the processor, preventing usage; trigger errors in machine microcode or sequencing of instructions, forcing the computer into an unstable state; exploit operating system vulnerabilities to sap system resources; crash the operating system altogether. The overriding similarity in these examples is that, as a result of the successful Denial of Service attack, the system in question does not respond as before, and service is either denied or severely limited.[9]

\subsection{Sources of Denial of Service Attacks}

DoS attacks are low-cost, and difficult to counter without the right tools. This makes them highly-popular even for people with technical knowledge. In fact, DoS services are offered on some web sites starting at $\$ 50$. These services have grown more and more sophisticated, and can effectively exploit application vulnerabilities and evade detection by firewalls.

According to market research, DoS attacks largely originate from people with a grudge or complaint against a web site or company, competitors looking to increase market share by damaging commercial web availability, or criminal elements that systematically extort web site owners by holding his assets for ransom. [9]

DENIAL OF SERVICE (DoS) attacks [1] are very common in the world of internet today. Increasing pace of such attacks has made servers and network devices on the internet at greater risk than ever before. Due to the same reason, organizations and people carrying large servers and data on the internet are now making greater plans and investments to be secure and defend themselves against a number of cyber attacks including Denial of Service. The traditional architecture of World Wide Web is vulnerable to serious kinds of threats including DoS attacks. The attackers are now quicker in launching such attacks because they have sophisticated and automated DoS attack tools available which require minimal human effort. The attack aims to deny or degrade normal services for legitimate users by sending huge traffic to the victim (machines or networks) to exhaust services, connection capacity or the bandwidth. In figure 1, five types of DoS attacks are mentioned. In network device level attacks, the target is some hardware device on the network such as a router. The attack is launched by exploiting some software bug or hardware resource vulnerability. In Operating System (OS) level attacks, vulnerabilities of operating system in the victim machine are used to launch DoS attack. In application level attacks, bugs or vulnerabilities in the application are identified to exploit them for DoS attack. Port scanning for identifying open ports of a remote application is very common in this perspective. Such attacks are now getting more popular as they present the traffic to a network and its devices similar to the legitimate traffic. Therefore, in a scenario where most of other attacks are now identifiable, application level attacks offer more success rate to attackers. In data flood attacks, targets are the connection capacity of a remote host or the bandwidth of a network. Heavy traffic is generated by the attacker towards the victim to exhaust connectivity or bandwidth resources so that normal services are denied or degraded for requests of 
legitimate users. In protocol feature attacks, the weaknesses of some protocol features are used to exploit them for launching a DoS attack. For example, the source IP address of a data packet (which relates to Internet Protocol and is a part of TCP/IP stack) can be spoofed by an attacker to launch a DoS attack which can be harder to trace due to a fake address [6].

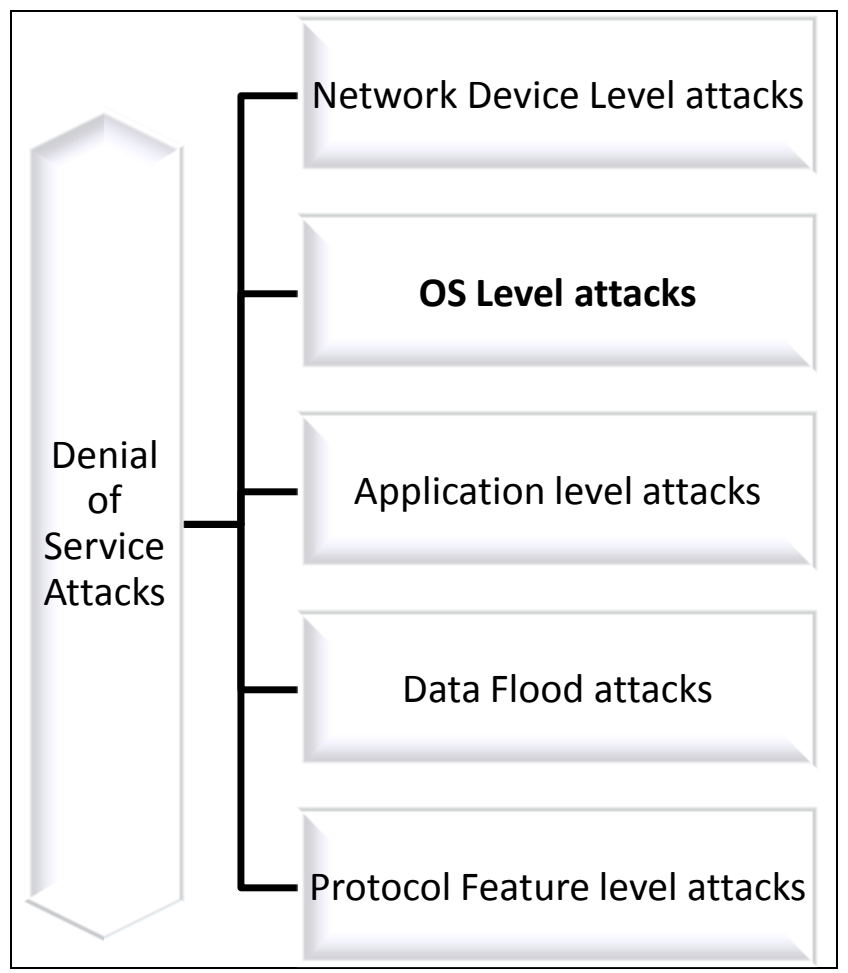

Figure 1: Denial Service Attacks [6]

\subsection{Distributed Denial of Service \\ Attacks}

In a Distributed Denial of Service (DDoS) attack, the attacker makes a huge impact on the victim by having multiplied power of attack derived by a large number of computer agents. It is made possible by the attacker through making a large number of computer machines under his control over the internet before applying an attack. In fact, these computers are vulnerable in the public network and the attacker exploits their weaknesses by inserting malicious code or some other hacking technique so that they become under the control of the attacker. These compromised machines can be hundreds or thousands in numbers. They behave as agents of the attacker and are commonly termed as 'zombies'. The entire group of zombies is usually named as a 'botnet'. The size of the botnet decides the magnitude of attack. For larger botnet (increased number of zombies in a botnet), attack is more severe and disastrous. Within a botnet, the attacker chooses 'handlers' which perform command and control functions and pass the instructions of the attacker to the zombies. The zombies directly attack on the victim. There is a group of zombies or agents under each handler. These handlers also pass the information received from zombies about the victim to the attacker. Therefore, handlers are the machines which directly communicate with the attacker and zombies. As the handlers and zombies are also compromised machines in such machines are usually unaware of the fact that there machines are being used as a part of some botnet. A typical architecture of DDoS attack is mentioned in figure 2.

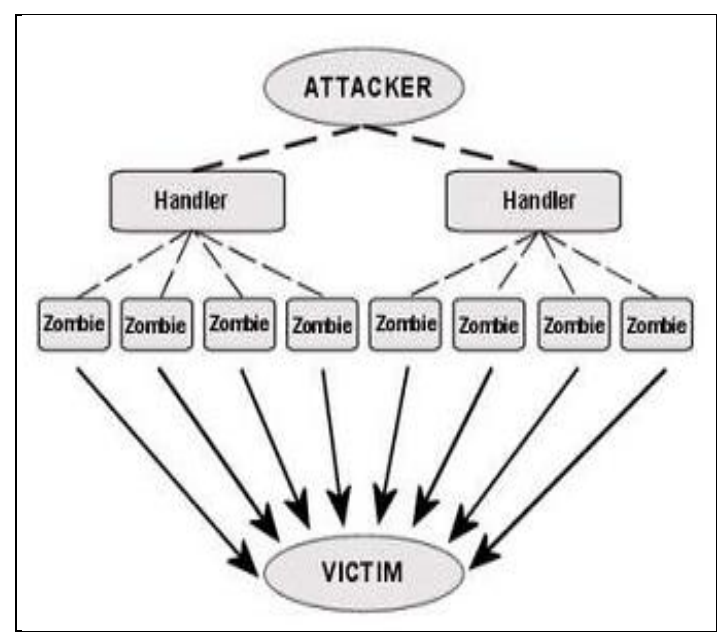

Figure 2: Architecture of DDoS attack [8]

The attack employs client server technology and a stream of data packets is sent to the victim for exhausting its services, connections, bandwidth etc. The data flood attack type of DoS is mostly used in DDoS attacks .DDoS attacks are further classified by attack rate dynamics i.e. the way how rate of attack varies with respect to the passage of time. The classes are Continuous Rate and Variable Rate attacks. In continuous rate, the attack has constant flow after it is executed. On the other hand, variable rate attack changes its impact and flow with time, making it more difficult to detect and respond. Within variable rate, the attack rate dynamics can further be implemented as Fluctuating or Increasing. Moreover, based on the data rate of attack traffic in a given network, the attacks are also categorized as high rate and low rate DDoS attacks [2] .DDoS attacks are also classified in the literature as "by impact' i.e. it can be Disruptive in which the normal service is completely unavailable to users, or it can be Degrading in which the in the productivity.

\subsection{Difference between DoS and DDoS Attack}

It is important to differentiate between Denial of Service (DoS) and Distributed Denial of Service (DDoS) attacks. In a DoS attack, one computer and one internet connection is used to flood a server with packets, with the aim of overloading the targeted server's bandwidth and resources. DDoS attack, uses many devices and multiple Internet connections, often distributed globally into what is referred to as a botnet. A DDoS attack is, therefore, much harder to deflect, simply because there is no single attacker to defend from, as the targeted resource will be flooded with requests from many hundreds and thousands of multiple sources.

\section{SOME COMMON DDOS ATTACKS}

\subsection{Direct and Reflector Attacks}

In direct DDoS attacks, zombies directly attack the victim as shown in Figure 2. On the other hand, in reflector attacks, zombies send request packets with spoofed IP (IP of victim) in source address field of IP packets to a number of other vulnerable computer devices (PCs, routers etc.) and replies generated from such devices are routed towards the victim for an impact desired by attacker. In such a way, reflection of the traffic is seen in these attacks. A classic example is sending "ping" requests with spoofed source IP. In such a case, "ping" replies are sent towards victim. In this way, the attacker is 
successful in saturating victim's bandwidth. In direct DDoS attacks, attacker proceeds with instructions to „Handlerse which perform Command \& Control operations to control zombies. Moreover, zombies directly attack victims and also pass the information to handlers. In reflector attacks, modus operandi is almost the same but zombies further exploit reflectors (machines on targeted victim's network) to flood victim with huge amount of traffic (IP packets).

\subsection{Application Layer DDoS Attacks}

Since DDoS attacks are very old technique, there have been many researches and implementations to counter such attacks. Many forms of DDoS attack detection and mitigation are now available. However, the major focus of attackers in earlier times has been towards exhausting victim's services for legitimate users through network layer (layer 3) attacks i.e. modifying IP packet fields or flooding victim's network with data packets. However, as many defenses are now available against such attacks, attackers have also changed their strategies and started focusing on attacks of application layer (layer 7). In such attacks, no manipulation is done in IP packets on network layer level; instead, complete TCP connections are made with victim just like legitimate clients. After establishment of successful connections, attackers exhaust server (the victim) with requests of heavy processing for longer times (for instance, heavy image downloading is requested). In this way, server remains busy to process

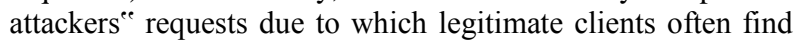
their requests unanswered.

Since complete TCP connections are made with servers in case of application layer attacks, such attacks are very difficult to identify and mitigate as normal traffic and attacking traffic are the same at network layer. Therefore, many traditional DDoS detection schemes fail in case of application layer DDoS attacks. Due to the same reason, researchers have also made several attempts in last few years to detect \& mitigate application layer DDoS attacks.

In table 1, different forms of common DDoS attacks in network and application layers are mentioned. Figure 3 and Figure .4 depict normal TCP three-way handshake operation and TCP ACK attack formation respectively.

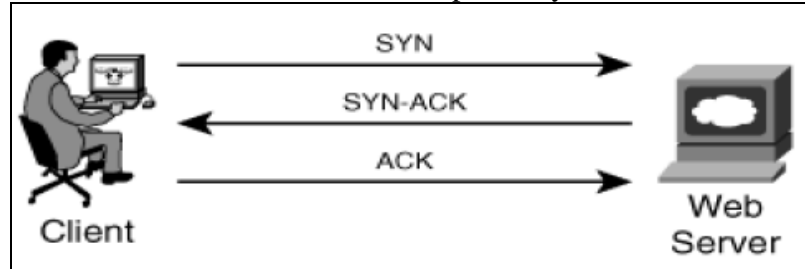

Figure 3: Three-way handshake in TCP [8]

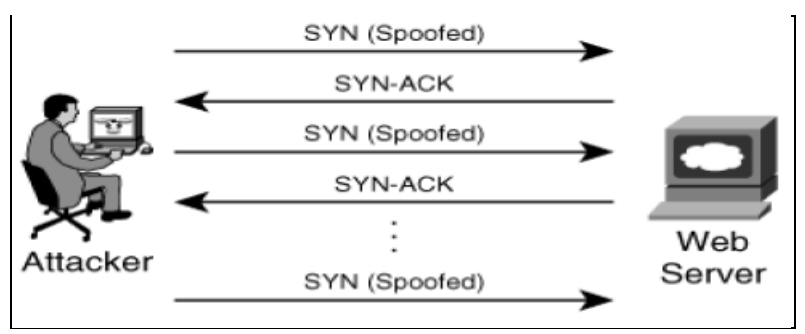

Figure 4: SYN ACK attack in TCP [8]

\subsection{Network Layer DDoS Attacks}

In the network layer or infrastructure layer (Layer 3) attacks, the malicious part resides in packet header or payload to compromise victim's CPU cycles, processing, bandwidth etc. However, with the introduction of sophisticated DDoS detection \& mitigation tools, attackers have also started changing their strategies to avoid detection and mitigation by increasing their focus towards application layer (Layer 7) attacks. These attacks mimic the legitimate clients to disturb or destroy the victim's resources. Therefore, traditional DDoS detection techniques are unable to identify such attacks. In these attacks, complete communication with the victim is established just like legitimate users. However, numerous connections are generated aiming to deny or degrade the service or bandwidth for legitimate clients. Application layer attacks are subject to the establishment of complete TCP connections with the victim. Therefore, the attacker has to disclose real IPs of zombie machines to the victim. Otherwise, it is not possible to make such connections. However, due to large number of zombies, the attacker does not worry about this attack limitation [3]. If such machines are identified and filtered at some stage, the attacker uses other group or pool of zombies to process the continuity of the attack. After establishing TCP connections with the victim in a large number, the attacker starts communication through sending requests for relatively large processing such as downloading heavy image files or making database queries. In this way, resources are reserved against such attack traffic to deny or degrade the services for legitimate users. Effectively, application layer attacks are also flooding attacks and categorized as HTTP flood, HTTPS flood, FTP flood etc. Sometimes, they are collectively mentioned as GET floods.

Table 1: Different forms of common DDoS attacks in network and application layers [10]

\begin{tabular}{|c|c|c|c|}
\hline Layer & Attack & Method & Impact \\
\hline \multirow[t]{3}{*}{$\begin{array}{l}\text { Network } \\
\text { Layer }\end{array}$} & $\begin{array}{l}\text { UDP } \\
\text { Flood }\end{array}$ & $\begin{array}{l}\text { Sending huge } \\
\text { amount of UDP } \\
\text { packets towards } \\
\text { victim's } \\
\text { bandwidth. }\end{array}$ & $\begin{array}{l}\text { Network } \\
\text { Congestion due } \\
\text { to } \\
\text { unavailability } \\
\text { of bandwidth to } \\
\text { legitimate } \\
\text { clients. }\end{array}$ \\
\hline & $\begin{array}{l}\text { ICMP } \\
\text { Flood }\end{array}$ & $\begin{array}{l}\text { Sending huge } \\
\text { amount of ICMP } \\
\text { packets towards } \\
\text { victim's } \\
\text { bandwidth. }\end{array}$ & $\begin{array}{l}\text { Network } \\
\text { Congestion due } \\
\text { to } \\
\text { unavailability } \\
\text { of bandwidth to } \\
\text { legitimate } \\
\text { clients. }\end{array}$ \\
\hline & $\begin{array}{l}\text { TCP } \\
\text { Flood }\end{array}$ & $\begin{array}{l}\text { Initiating large } \\
\text { number of TCP } \\
\text { connections (of } \\
\text { spoofed packets) } \\
\text { with victim and } \\
\text { not } \\
\text { acknowledging } \\
\text { the same (known } \\
\text { as TCP ACK } \\
\text { attack) }\end{array}$ & $\begin{array}{l}\text { Unanswered } \\
\text { Requests due to } \\
\text { unavailability } \\
\text { of connections } \\
\text { for legitimate } \\
\text { clients } \\
\text { (Connection } \\
\text { buffer i.e. } \\
\text { capacity is } \\
\text { limited on a } \\
\text { given server). }\end{array}$ \\
\hline $\begin{array}{l}\text { Application } \\
\text { Layer }\end{array}$ & $\begin{array}{l}\text { HTTP } \\
\text { Flood }\end{array}$ & $\begin{array}{l}\text { Establishing } \\
\text { large number of } \\
\text { TCP connections } \\
\text { with victim and } \\
\text { sending requests } \\
\text { for heavy }\end{array}$ & $\begin{array}{l}\text { Unanswered } \\
\text { Requests due to } \\
\text { unavailability } \\
\text { of server's } \\
\text { processing } \\
\text { cycles for }\end{array}$ \\
\hline
\end{tabular}




\begin{tabular}{|c|c|c|}
\hline & $\begin{array}{l}\text { processing } \\
\text { through HTTP } \\
\text { communication. }\end{array}$ & $\begin{array}{l}\text { legitimate } \\
\text { clients (All } \\
\text { processing } \\
\text { remains busy } \\
\text { for answering } \\
\text { attacker's } \\
\text { requests of } \\
\text { heavy } \\
\text { processing). }\end{array}$ \\
\hline $\begin{array}{l}\text { HTTPS } \\
\text { Flood }\end{array}$ & $\begin{array}{l}\text { Establishing } \\
\text { large number of } \\
\text { TCP connections } \\
\text { with victim and } \\
\text { sending requests } \\
\text { for heavy } \\
\text { processing } \\
\text { through HTTPS } \\
\text { communication. }\end{array}$ & $\begin{array}{l}\text { Unanswered } \\
\text { Requests due to } \\
\text { unavailability } \\
\text { of server's } \\
\text { processing } \\
\text { cycles for } \\
\text { legitimate } \\
\text { clients (All } \\
\text { processing } \\
\text { remains busy } \\
\text { for answering } \\
\text { attacker's } \\
\text { requests of } \\
\text { heavy } \\
\text { processing). }\end{array}$ \\
\hline $\begin{array}{l}\text { FTP } \\
\text { Flood }\end{array}$ & $\begin{array}{l}\text { Establishing } \\
\text { large number of } \\
\text { TCP connections } \\
\text { with victim and } \\
\text { sending requests } \\
\text { for heavy } \\
\text { processing } \\
\text { through FTP } \\
\text { communication. }\end{array}$ & $\begin{array}{l}\text { Unanswered } \\
\text { Requests due to } \\
\text { unavailability } \\
\text { of server's } \\
\text { processing } \\
\text { cycles for } \\
\text { legitimate } \\
\text { clients (All } \\
\text { processing } \\
\text { remains busy } \\
\text { for answering } \\
\text { attacker's } \\
\text { requests of } \\
\text { heavy } \\
\text { processing). }\end{array}$ \\
\hline
\end{tabular}

\subsubsection{DDoS Attacks in Wireless Networks}

Wireless networks are vulnerable to many kinds of attacks including distributed denial of service attacks. Their main vulnerability is shared wireless medium due to which many attacks are possible to exploit and compromise wireless stations. It is possible in almost all variations of wireless networks such as Wireless sensor networks (WSN), Mobile ad hoc networks (MANET) and Wireless local area networks (WLAN) [2-5]. Like traditional wired networks, DDoS attacks on wireless networks are also possible in different layers of communication. Some common forms of DDoS attacks in different layers of wireless networks are indicated in table 2 .

Table 2: some common forms of DDoS attacks in different layers of wireless networks [10]

\begin{tabular}{|l|l|}
\hline Layer & Attack \\
\hline Physical Layer & Jamming Attack \\
\cline { 2 - 2 } & Node Tampering Attack \\
\hline \multirow{2}{*}{ Link/MAC Layer } & Interrogation Attack \\
\cline { 2 - 2 } & Collision Attack \\
\hline \multirow{2}{*}{ Network Layer } & Black Hole Attack \\
\cline { 2 - 2 } & HELLO Flood Attack \\
\hline Transport Layer & SYN Flooding Attack \\
\hline Application Layer & Overwhelming Attack \\
\hline
\end{tabular}

DoS Attack (Path Based)

\section{SYSTEM MODEL}

\subsection{Approaches for solving the problem}

\subsubsection{Detection Architecture}

The general technique of this recognition construction modeling is shown in Fig. The plan is partitioned into three stages:

Login: Login table containing username and secret key

Access: contains data (like username, secret key, and IP address) about all customers who got to the particular site for quite a while of time.

Administrator log: This table containing username and secret key

Peruse log: This table containing full customer skimming purposes of investment like customer who looked, count log, start time, end time, site address, system name and date.

Administration: This table containing server IP address, client name, record size and fcount.

Srm: This table containing ID and name to be shown.[7]

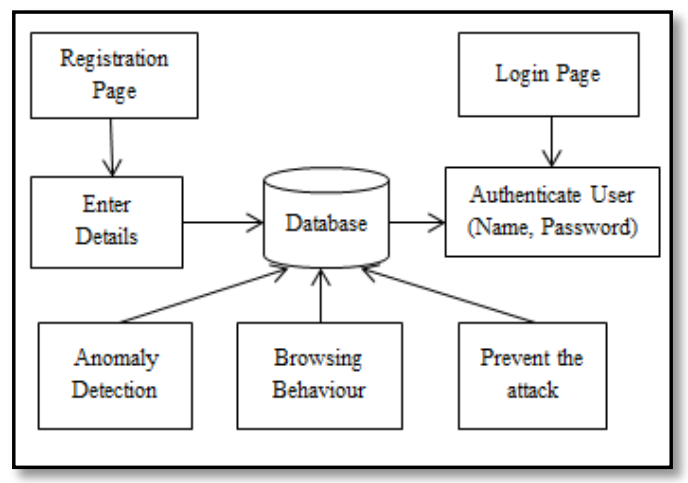

Figure 5: Detection Architecture[7]

\subsubsection{Login/Registration}

The Valid consumer get into login to send data to accessible system frameworks, if the consumer does not enlist it'll move to new consumer creation from. During this Module assembling the final consumer points of interest and store info for future references.

It is having Name, Password, ensure positive identification, and Email address.

\subsubsection{Anomaly Detection}

Inconsistency recognition depends on identifying practices that are unusual regarding some typical standard. Numerous peculiarity recognition frameworks and methodologies have been created to identify the weak indications of DDoS assaults.

\subsubsection{Browsing Behavior}

Inconsistency recognition depends on identifying practices that are unusual regarding some typical standard. Numerous peculiarity recognition frameworks and methodologies have been created to identify the weak indications of DDoS assaults.

\subsubsection{Prevent the Attack}

By the utilization of a DDoS instrument the source IP location of the assaulting parcels can be mock and thusly the genuine character of the optional victimized people is kept from presentation and the return bundles from the exploited person framework. At that point preclude the entrance from claiming the clients. [7] 


\subsection{Facade Layer}

Definition for facade vogue pattern is, "Give a sure along interface to a group of interfaces terribly exceptionally topic. Exterior Pattern characterizes a additional elevated quantity interface that makes the topic easier to use." to modify the affiliation approach, we have a tendency to tend to possess an inclination to gift veneer layer. Veneer uncovered academic degree improved interface (for this instance one interface to perform that multi-step process) and inside it connects with those segments and gets the embody strait you. It'll be taken joined level of reflection over partner existing layer.

Facade style pattern is one in all the opposite configuration styles that advance detached coupling. It accentuates another essential a part of configuration that is deliberation. By concealing the many-sided quality behind it and uncovering a basic interface it attains to deliberation

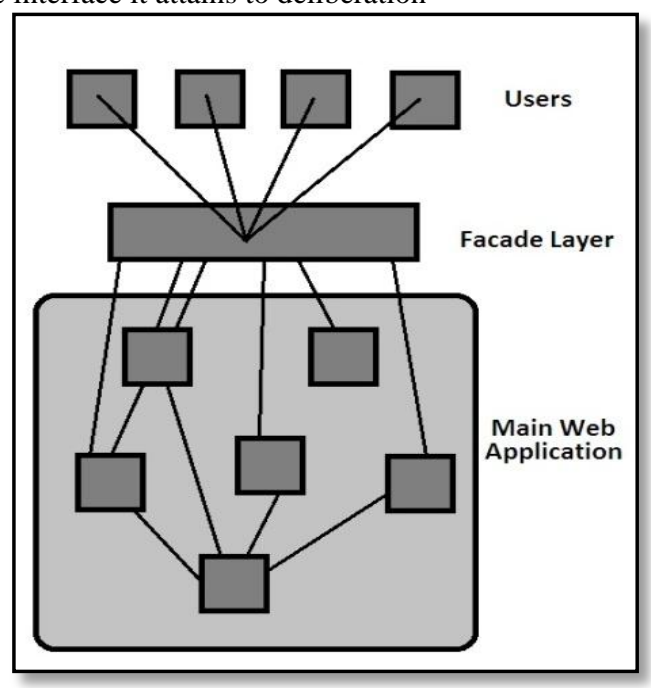

Figure 6: FAÇADE Layer

\subsection{Application Layer}

Application layer is that the most astounding layer in OSI and TCP/IP stratified model and, this layer exists in each stratified models owing to its importance that is collaborating with consumer and consumer applications. This layer is for applications that square measure enclosed in correspondence framework.

A consumer might probably foursquare collaborate with these applications. Application layer is that the place the important correspondence is launched and reflects. Since this layer is on the very best purpose of the layer stack it does not serve no matter alternative layers. Application layer takes the help of transport and every one layers below it to convey or exchange its info to the remote host.

At the purpose once associate application layer convention has to speak with its associate application layer convention on remote hosts it hands over the knowledge or data to the Transport layer.

The vehicle layer will no matter is left of the items with facilitate of all layers below it.

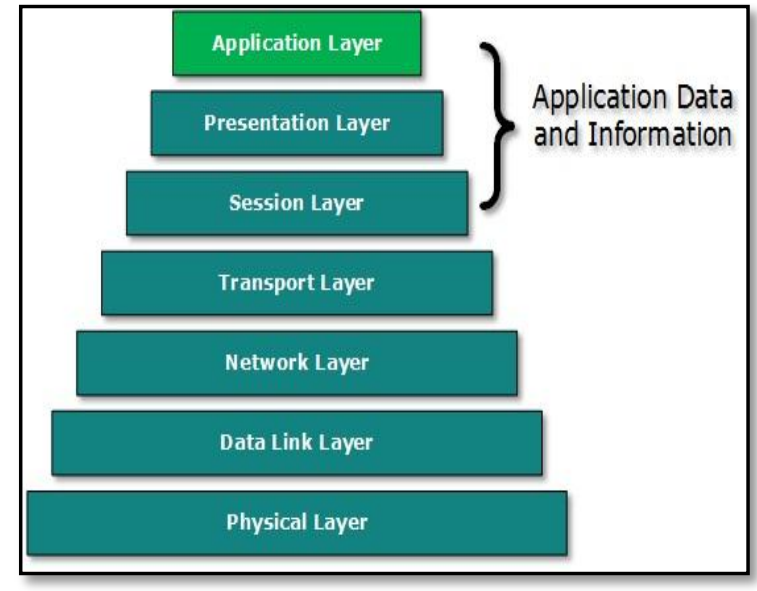

Figure 7: Application Layer

There is ambiguity in understanding Application Layer and its convention. Not each consumer application will be place into Application Layer. Simply application that interfaces with the correspondence framework. For example, associate outlining programming or word processing system cannot be thought of as application layer comes.

Then again after we utilize an internet Browser that is basically utilizing protocol (Hyper Text Transfer Protocol) to interface with the system. Thus for this case, protocol is Application Layer convention that we tend to ponder after we study superimposed models.

An alternate illustration is File Transfer Protocol that helps a consumer to exchange a content based mostly or parallel document over the system. A consumer will utilize this convention as a region of either GUI based mostly programming like File Zilla or Cute FTP and also the same consumer will utilize FTP as a region of statement mode.

So it's not imperative what programming you utilize, it's the convention that is taken into account at Application Layer used by that product. DNS may be a convention that helps consumer application conventions like protocol to perform its work.

\subsection{System Architecture}

Old system was not capable to detect attack on application layer, comparatively new system can detect attack in new sub layer of application layer named "FACADE" layer.

Attack detection was not available for web services which are made possible by new system. New developed system is detachable from main application working behind. Developed system can manage user sessions which makes main application completely service oriented.

The key features of the proposed work are:

(1) Multiple checks happen over client.

(2) Main application can be kept safe and separate from attacks because of facade.

(3) False rejection rate is very low.

(4) The facade box is easily attachable and detachable to main application.

Comparing with the normal user requests, In DDoS the request rate increases significantly in very short time. The proposed system in facade layer has two phases, in first analyzing of the available data about the user and its characteristics takes place. Using that analysis a score is assigned to the each user. Then the entropy of requests per 
session is calculated. Entropy is an information theoretical concept, which is a measure of randomness. The entropy is employed in this paper to measure changes of randomness of requests in a session for a given time interval. Then, based on the request history the user the trust score is assigned to the user.

In second phase, detection of the DDoS attack takes place. The entropy for the current session is calculated and degree of deviation with the predefined value is estimated. The amount of the deviation decides how suspicious the user is. Greater the degree of the deviation more the user is suspicious. The rate limiter, Scheduler and request blocker is also there in facade layer. Rate limiter sets proper thresholds and limits, based on which filtering is happen. Scheduler schedules the buffered requests on the basis if the server workload. Figure 6.1 demonstrates the inside outline of the exterior layer. The recognition instrument is moreover conveyed in veneer. The validation appeal comes first to the veneer, then the exteriors show that client if legitimate accreditations square measure gave by returning him the authentication key. In the event that client is asking for confirmation yet again still indistinguishable authentication mysteries gave to him, this system keeps him in same session and keeps him from making new session.

On the off chance that the deviation is at interims limit, then the pace circuit channels the session upheld the trust score of the client. The customer UN office carries on higher in history can get higher level of trust. In the event that the client is considered authentic, and then the PC equipment plans the appeal backed the occupation of the framework. The absolute best trust score beginning arrangement is utilized to calendar the solicitations for the server.

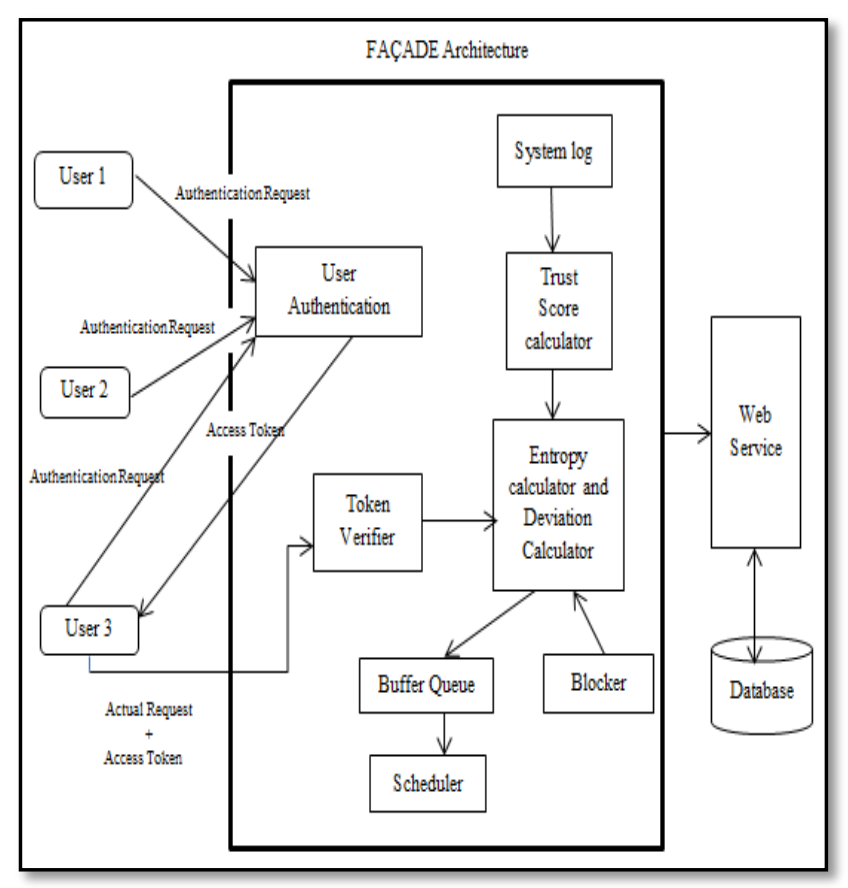

Figure 8: System Architecture

\subsubsection{Entropy Calculation}

Let the charming quality in associate passing session be showed as $r_{i j}$, where $i, j$, a get-together of positive whole numbers. "i" infers the posing for mixture in session ' $j$ '. Let | $\left(r_{j}, t\right) \mid$ hint the live of offers every session $j$, at a given time $t$. Around then,

$$
\left|\left(\mathrm{r}_{j}, \mathrm{t}\right)\right|=\sum_{i=l}^{\infty} r_{i j}
$$

For a given interim $\Delta t$, the range within the amount of appeals each session $\mathrm{j}$ is given as takes when

$$
N_{j}\left(r_{j}, t+\Delta t\right)=\left|r_{j}, t+\Delta t\right|-\left|\left(r_{j}, t\right)\right|
$$

The probability of the requests per session $\mathrm{j}$, is given by

$$
P_{j}\left(r_{j}\right)=N_{j}\left(r_{j}, t+\Delta t\right) / \sum_{i=l}^{\infty} \sum_{j=l}^{\infty} N_{j}\left(r_{j}, t+\Delta t\right)
$$

Let $\mathrm{R}$ be the capricious variable of the live of advances every session amidst the between time $\Delta t$, afterwards, the entropy of requesting every session is given:

$$
H(R)=-\sum_{j} P_{j}\left(r_{j}\right) \log P_{j}\left(r_{j}\right)
$$

In light-weight of the qualities of entropy limit, the upper and farthest purpose of the entropy $\mathrm{H}(\mathrm{R})$ is delineate as

$$
0 \leq H(R) \leq \log N
$$

Where $\mathrm{N}$ is the number of the requests.

Under DoS ambush, the number of provide extends through and thru and also the going hand in hand with correlation holds

$$
|H(R)-C|>\text { threshold, } t
$$

Where $\mathrm{C}$ is the maximum capacity of the session. [7]

\subsubsection{Rate Limiter}

To keep expelled from erroneously disclosure, rate-limiter is given. Once the entropy is dead set, enlist the degree of deviation from the predefined entropy. The structure first sets a farthest point for acceptable deviation. Within the event that the registered deviation surpasses the sting, then the session is compelled to finish quickly. one thing else, second level channel is connected by the speed electric circuit. The framework to boot characterizes a footing for approving a shopper taking into consideration the trust score. A shopper is believed to be true blue simply if the trust score surpasses the limit. one thing else, the shopper is viewed as malevolent and also the session is born quickly. The sessions area unit then gone to the computer hardware for obtaining administration from the server. [7]

\subsubsection{Scheduler}

In the event that the consumer is real, then the computer hardware plans the session taking under consideration the foremost lowest suspicion initial (client with most noteworthy trust score) arrangement. The in good order carried on shoppers can have Associate in Nursing nearly no deviation. In such case, the authentic consumer gets a quicker administration. Withal the look approach, framework employment is to boot thought of before booking the attractiveness for obtaining administration. [7]

\subsubsection{Monitoring Algorithm}

Input: system log:

1. Extract the solicitation entries for all sessions, page survey time and therefore the arrangement of asked for things for each consumer from the framework $\log$. Compute the entropy of the requests per session victimization the formula: 


$$
H_{\text {new }}(R)=-\sum_{j} P_{j}\left(r_{j}\right) \log P_{j}\left(r_{j}\right)
$$

2. Work out the trust score for each single consumer taking under consideration their review time and planning to conduct.

\subsubsection{Detection Algorithm}

Data the predefined entropy of solicitations each session and therefore the trust score for each consumer. Characterize the sting connected with the trust score (Tts) outline the limit for cheap deviation (Td) for each session holding up for discovery Extract the appeals landings Figure the entropy for each session utilizing (4)

$$
H_{\text {new }}(R)=-\sum_{j} P_{j}\left(r_{j}\right) \log P_{j}\left(r_{j}\right)
$$

Compute the degree of deviation:

$$
D=\left|H_{\text {new }}(R)\right|-|H(R)|
$$

$n$ the off likelihood that the extent of deviation isn't precisely the permissible edge (Td), and client's trust score is additional noteworthy than the limit (Tts), then allow the session to induce administration from the online server.

The session is pernicious; drop it. [7]

\section{IMPLEMENTATION RESULTS}

The implementation is done in JAVA and results of the experiments are explained in this section. Snapshots of simulation work done have explained.

The snapshots are divided according to the processing and are:

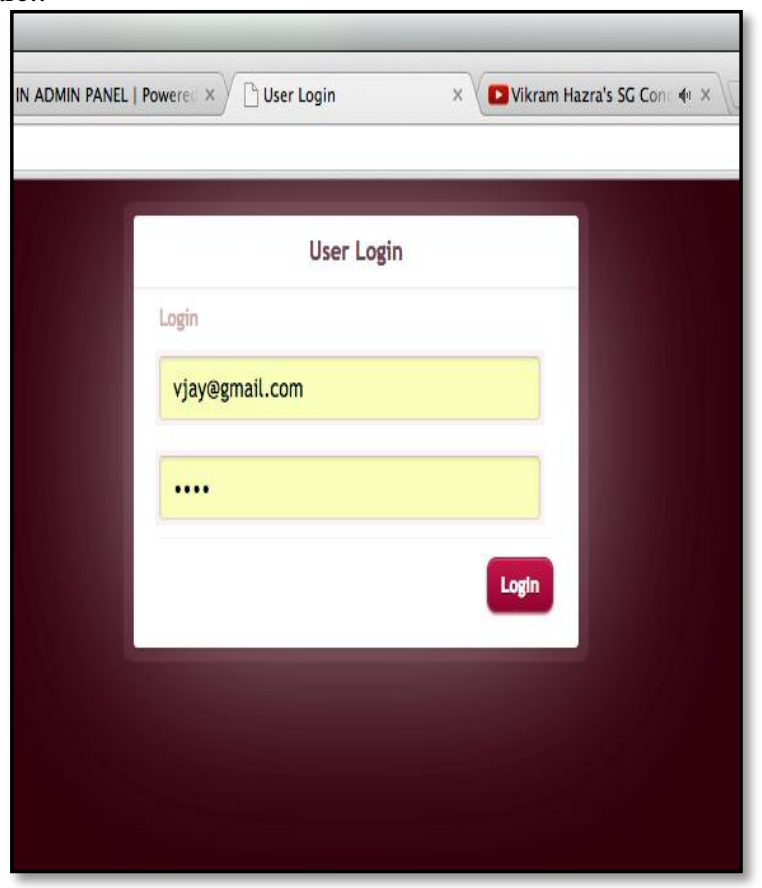

Figure 9: Admin Window (New user request for accessing application)

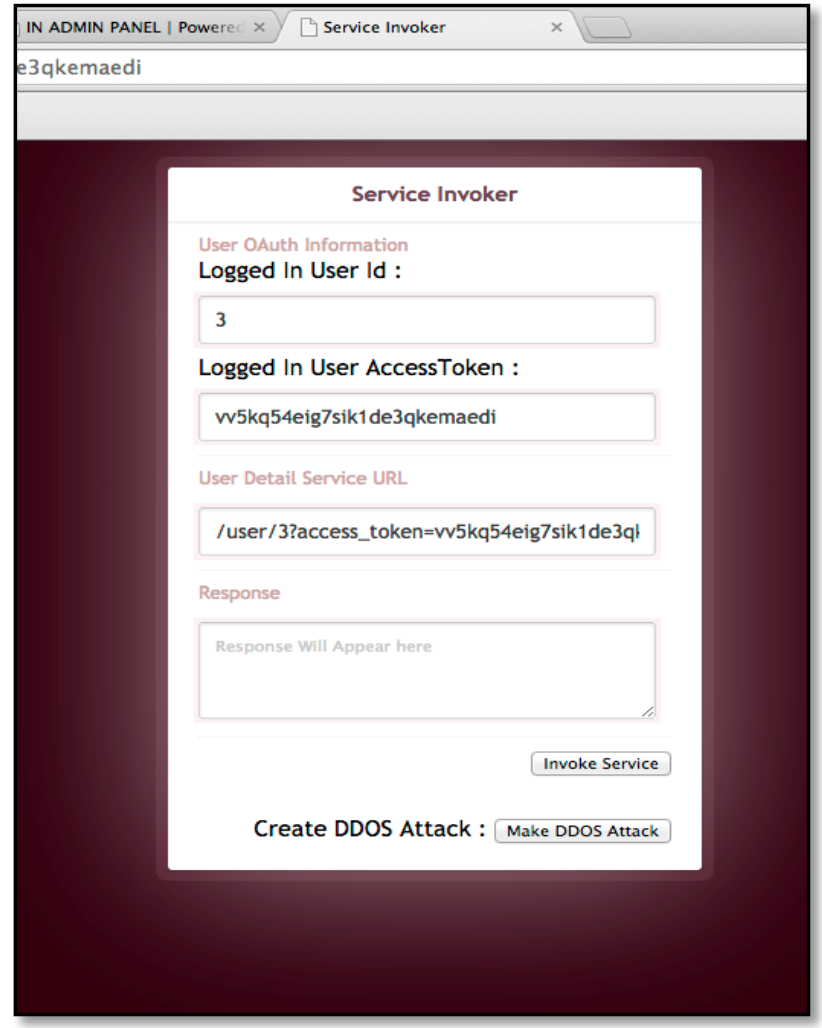

Figure 10: New user assigned with token for accessing web services

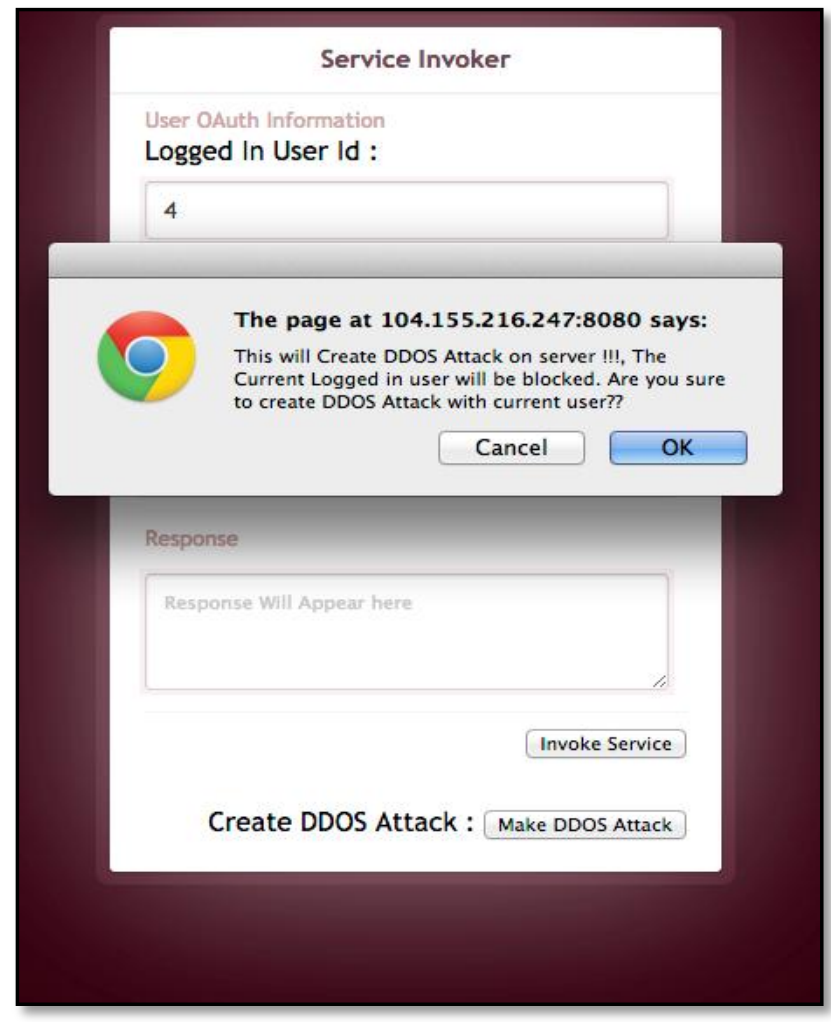

Figure 11: Admin window showing the user is attacker and will blocked if processed 


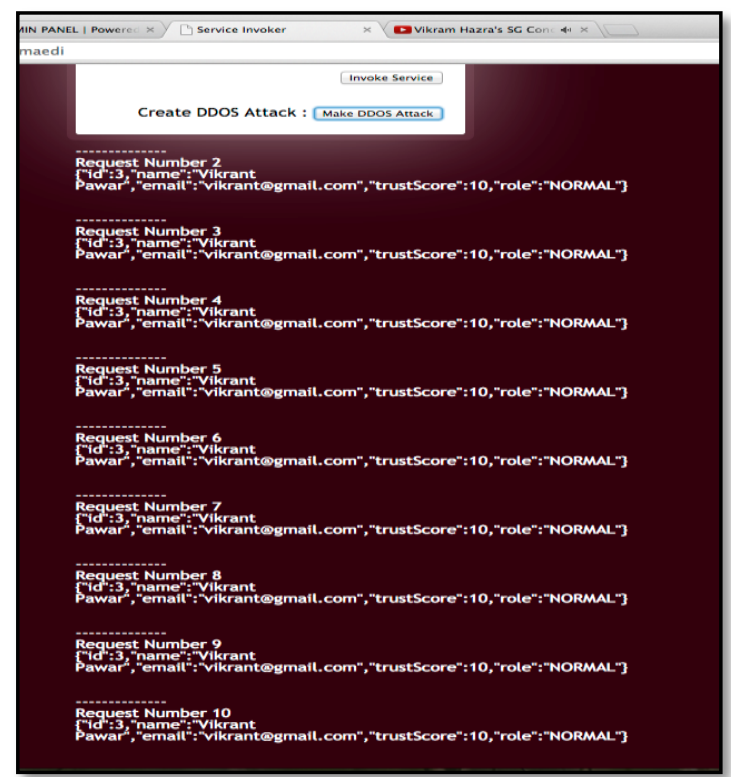

Figure 12: New user request accessed by system till Trust score (For our system Trust score $=10$ )

When the new user requests for permission to access the web service, the server always calculates trust score for that user by following formula:

$$
\text { Trust Score }=\sum_{\substack{s=0 \\ \text { Where, } \mathrm{s}=\text { number of sessions }}}^{n} \frac{\text { number of requests in current session }}{\text { number of sessions }}
$$

So for this session, maximum numbers of requests are 20 and sessions are 2. So, the trust score is 10 .

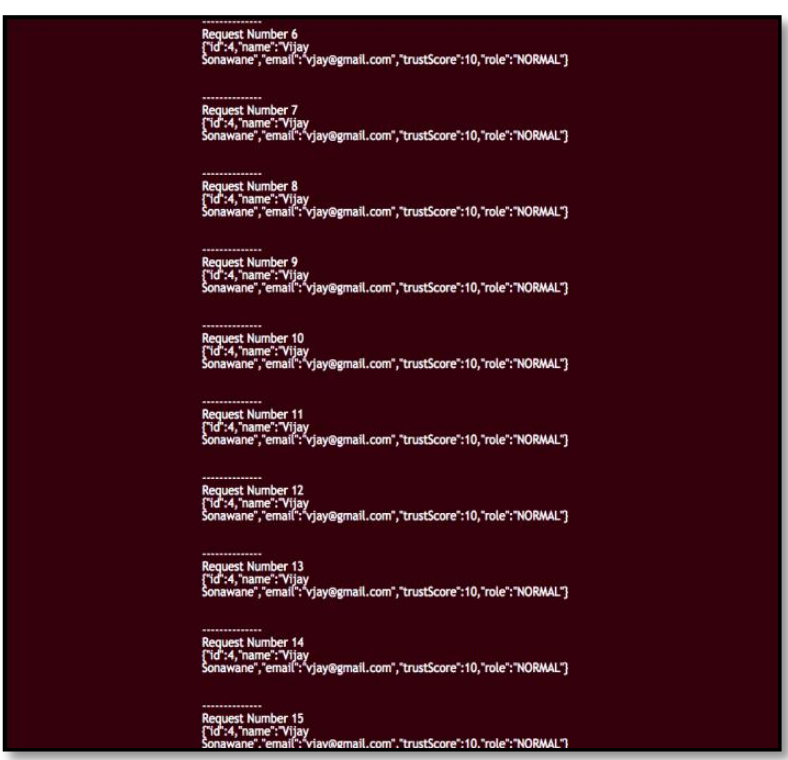

Figure 13: Request access by layer for new user till threshold value that is 15

Figure 13 shows that, sometimes, the user is genuine, that is, due to some network problem or any other issue the requests may be repeated so the threshold value is considered. The threshold value here introduced is 5 as the user can make more 5 requests. The threshold value gives the genuine users a chance to access the service.

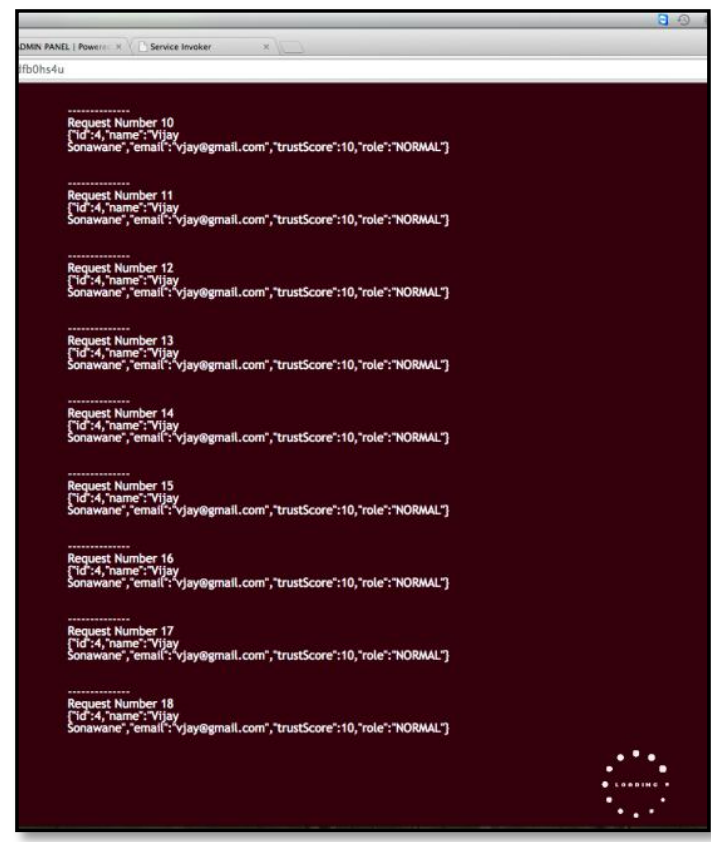

Figure 14: Request access by layer for new user till buffering value that is $\mathbf{1 5}$

(Trust score (10)+ threshold value (5) + Buffering value

Figure 14 shows that, sometimes, the user who after requesting again and again then the user goes in buffer. The buffer is used for collecting those users who are requesting continuously. The buffer value has taken 5 . When the buffer becomes active, the scheduler comes in working. So for more 5 requests the user will get to take permission for access.

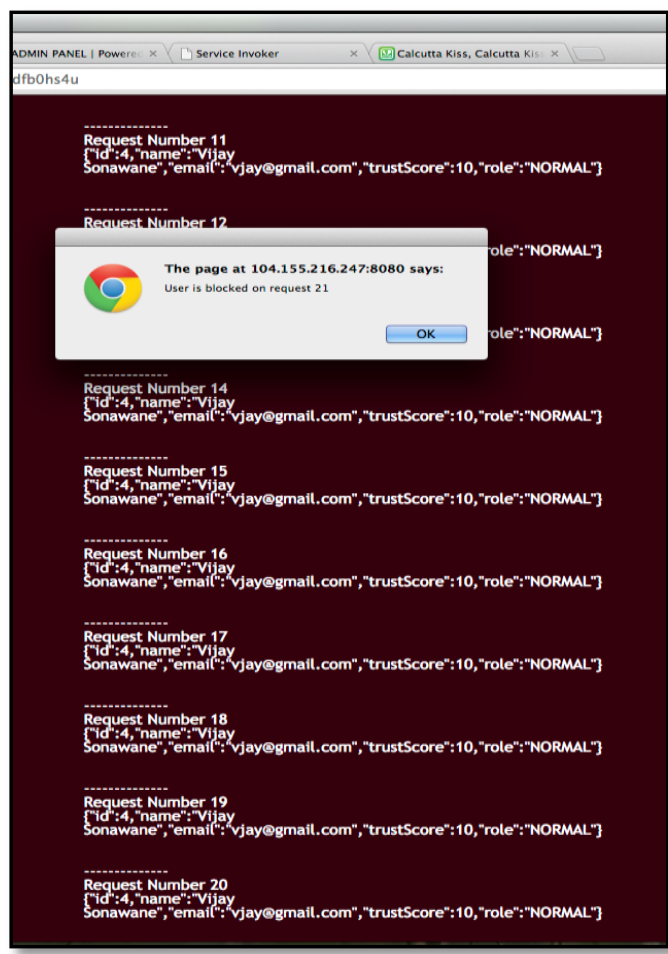

Figure 15: Next request for user after the limit that is after buffering will be blocked for next request 
Figure 15 shows that, after completing maximum allowed requests for the user that is the maximum number of requests required for accessing the web service for the next requests the user get blocked.

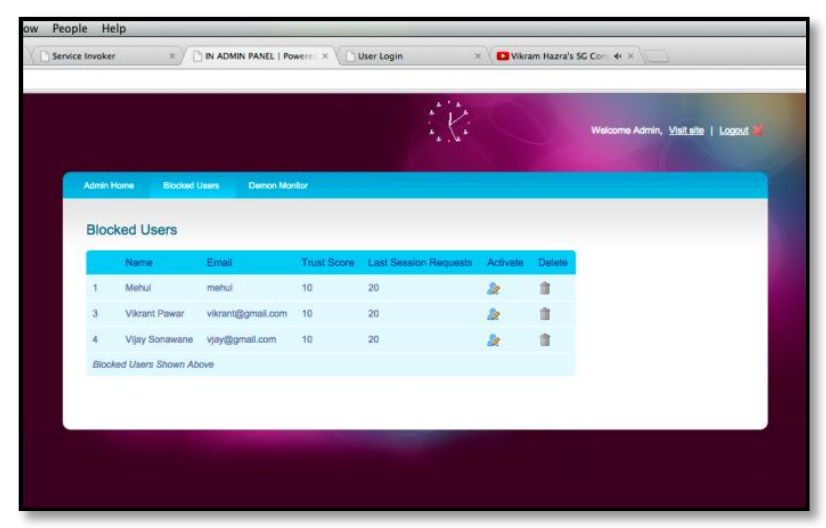

Figure 16: Admin window showing results for list of blocked user new blocked user added in list

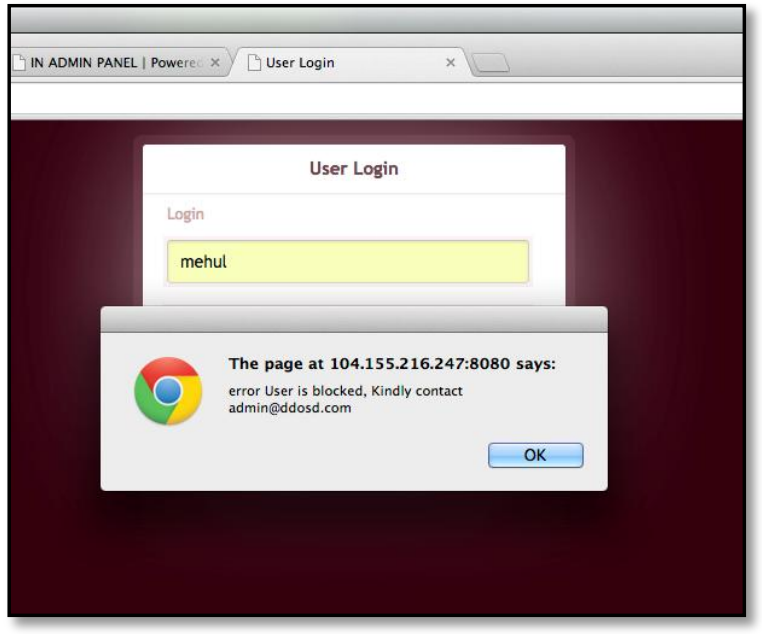

Figure 17: Admin window showing results for blocked user when it will log in

\section{CONCLUSION}

This paper proposes efficient way to track DDo'S attack over the REST (Representational State Transfer) web-services. Proposed way uses pre available information metric for existing users and starts monitoring new users immediately as well. Every request has to pass the multiple checks to reach to its web-service destination.

Developed application introduced efficient way, a new layer called FACADE to track DDoS attack as compared to the REST (Representational State Transfer) web-services. While REST stands For Representational State Transfer which is an architectural style for networked hypermedia applications, it is only used to build Web services that are lightweight, maintainable, and scalable only. The intermediate layer (FACADE) keeps the actual application totally isolated and away from user access areas. This application uses pre available information metric for existing users and starts monitoring new users immediately as well. Authentication for the requests is managed by highly encoded token service which is also part of proposed system. System also has a scheduler and rate limiter to downgrade the service to malicious user requests. Proposed system also has ability to block suspicious or malicious users. System provides workaround to traditional systems of DDoS detection and keeps trust level for individual user.

Such technique implementation has been on Web applications where server architecture is used. In future DDOS attacks will feature on portable systems as their computing power increases. Hence the proposed system will be required to be implemented on mobile \& tablets as well.

Secondly, the proposed system if combined with suitable hardware devices such as router or network controller, the security may be enhanced and for an effective defense may be established.

The cloud environment may also look at this mechanism as a service in future.

\section{ACKNOWLEDGMENTS}

Our thanks to the Prof. S. R. Todmal, Head of Computer Department, and to Principal, Dr. S. V. Admane for their valuable guidance in this research work.

\section{REFERENCES}

[1] J. B. D. Cabrera, L. Lewis, X. Qin, W. Lee, R. K. Prasanth, B. Ravichandran\& R. K. Mehra, "Proactive detection of distributed denial of service attacks using MIB traffic variables a feasibility study", in Proc. IEEE/IFIP Int. Symp. Integr. Netw. Manag., pp. 609-622 (2001).

[2] L. Limwiwatkul\& A. Rungsawangr, "Distributed denial of service detection using TCP/IP header and traffic measurement analysis," in Proc. Int. Symp. Commun. Inf. Technol., Sappoo, Japan,Oct. 26-29, pp. 605-610 (2004).

[3] S.Kandula, D.Katabi, MJacob\& A.W.Berger,"Botz-4sale: surviving organized DDoS attacks that mimic flash crowds", in Proc. Second Symp. Networked Systems Design and Implementation (NSDI) (2005).

[4] J. Yuan \& K. Mills, "Monitoring the macroscopic effect of DDoS flooding attacks," IEEE Trans. Dependable and Secure Computing, vol. 2, no. 4, pp. 324-335 (2005).

[5] W. Yen \& M.-F. Lee, "Defending application DDoS with constraint random request attacks," in Proc. Asia-Pacific Conf. Commun., Perth, Western Australia, pp. 620-624 (2005).

[6] Mitrokotsa, and C. Douligeris, "Denial-of-Service Attacks," Network Security: Current Status and Future Directions (Chapter 8), WileyOnline Library, pp. 117134, June 2006

[7] Ankali, Sanjay B., and D. V. Ashoka. "Detection architecture of application layer DDoS attack for internet." Int. J. Advanced Networking and Applications 3.01 (2011): 984-990.

[8] Aamir, Muhammad, and Mustafa Ali Zaidi. "DDoS Attack and Defense: Review of Some Traditional and Current Techniques." arXiv preprint arXiv:1401.6317(2014).

[9] Denial of Service Attacks, "http://www.incapsula.com/ddos/ddos-attacks/denial-ofservice.html"

[10] Sahu, SonaliSwetapadma, and ManjushaPandey. "Distributed Denial of Service Attacks: A Review." International Journal of Modern Education and Computer Science (IJMECS) 6.1 (2014): 65. 